\title{
Gastrointestinal Stromal Tumor (GIST) with Chondroid-Myxoid-Chordoid Features Mimicking Extraskeletal Myxoid Chondrosarcoma
}

\author{
Sonia Brar ${ }^{1}$, Christian Tvetenstrand ${ }^{2}$, Jagmohan Sidhu ${ }^{3}$ \\ ${ }^{1}$ Department of Medicine, UHS Hospitals, Johnson City, USA; ${ }^{2}$ Department of Surgery, UHS Hospitals, Johnson City, USA; \\ ${ }^{3}$ Department of Pathology and Laboratory Medicine, UHS Hospitals, Johnson City, USA. \\ Email: Jagmohan_sidhu@uhs.org
}

Received March $1^{\text {st }}, 2012$; revised April 1 ${ }^{\text {st }}, 2012$; accepted April $9^{\text {th }}, 2012$

\begin{abstract}
Epithelioid gastrointesinal tumors (GISTs) are less likely to have $c$-kit gene mutations (and express CD117) than spindle cell GISTs. CD117 negative/c-kit negative GISTs can have platelet-derived growth factor alpha (PDGFR $\alpha$ ) gene mutations, overexpress PDGFR a protein and respond to imatinib mesylate. Many cases of CD117-negative/CD117weakly positive, $c$-kit mutation negative and PDGFR $\alpha$ mutation positive myxoid epithelioid GISTs and one case of CD117-positive GIST with chondro-myxoid features mimicking chondrosarcoma have been reported. We report a case of myxoid epithelioid GIST with predominance of chondroid and chordoid areas resembling an extraskeletal myxoid chondrosarcoma that was strongly positive for CD117, PDGFR $\alpha$ and DOG1 (Discovered on GIST 1) by immunohistochemistry, but lacked c-kit and PDGFR $\alpha$ gene mutations. It is possible that CD117 is strongly positive if a myxoid epithelioid GIST has chondroid/chordoid appearance, but a larger study is needed to confirm this association. CD117 expression in GISTs is important, because GISTs showing CD117 positivity respond to imatinib. No comment can be made about the prognostic significance of chondroid/chordoid appearance in the GISTs.
\end{abstract}

Keywords: Gastrointestinal Stromal Tumor; GIST; PDGFR ; CD117; c-kit; Extraskeletal Myxoid Chondrosarcoma

\section{Introduction}

GISTs are the most frequent nonepithelial neoplasms of the gastrointestinal tract [1] and they are predominantly located in the stomach [2]. They arise from the interstitial cells of Cajal or their precursors, the gastrointestinal pacemaker cells [3]. Microscopically a majority of these tumors are composed of spindle or epithelioid cells and one or the other morphology usually predominates. Spindle cell or spindle cell-predominant GISTs usually show mutations in c-kit gene, are immunohistochemically positive for c-kit (CD117) and can be effectively treated with imatinib mesylate. Epithelioid GISTs sometimes do not show $c$-kit mutations and do not express CD117 [4,5]. In the literature, 27 cases of gastric epithelioid GISTs with myxoid change (without chondroid or chordoid appearance) have been described. Twenty (20) of these 27 gastric epithelioid GISTs with myxoid change were tested for CD117 expression by paraffin section immunohistochemistry, and were also tested for c-kit gene mutations and $P D G F R \alpha$ gene mutations. Sixteen (16) of 20 showed weak CD117 expression and 4 showed lack of CD117 expression. Twenty (20) of 20 cases showed lack of $c$-kit mutation and 18 of 20 cases showed presence of $P D G$ $F R \alpha$ gene mutation $[6,7]$. Therefore, myxoid change in a GIST has been reported to be associated with CD117 negativity or weak positivity. A single case of chondromyxoid variant of GIST mimicking chondrosarcoma with co-expression of CD117, CD99 and CD34 has been reported. CD117 expression in this a case was not called week. No mutational analysis was done in this case [8]. We report a case of myxoid epithelioid GIST with chondroid areas and chordoid areas that resembled an extraskeletal myxoid chondrosarcoma and strongly expressed CD117, PDGFRa, CD99, and DOG1 (Discovered on GIST 1) by immunohistochemistry, but was negative for CD34 and lacked mutations in the c-kit as well as $P D G-$ $F R \alpha$ gene. We also discuss the association of chondroid/ chordoid appearance in a myxoid GIST with strong CD117 positivity, which is important for chosing imatinib mesylate therapy if the need of this therapy arises.

\section{Materials and Methods}

The specimen was fixed in $10 \%$ buffered formalin and was embedded in paraffin. The histopathologic, immu- 
nohistochemical and mutation analysis for $c$-kit gene and $P D G F R \alpha$ gene was performed on this formalin-fixed paraffin-embedded specimen. Hematoxylin and Eosin stain, trichrome stain, alcian blue stain, and toluidine blue stain were performed. Immunohistochemical staining was done by using Ventana Ultra Analyzer (Ventana Medical Systems, Tucson, AZ, USA). Antibodies against CD117, CD34, CD99, S-100, desmin, smooth muscle actin, epithelial membrane antigen, pankeratin, DOG.1 (clone DOG1.1; dilution 1:100; Acris Antibodies, Inc. San Diego, CA), PDGFRa (clone 57/20; Abcam, Inc. Cambridge, MA), and D240 (clone D2-40; dilution 1:100; Covance, Emeryville, CA) were used, All antibodies, except DOG.1, PDGFR $\alpha$ and D2-40, were obtained from Ventana Medical Systems in Tucson, AZ. The clones and dilutions of the antibodies obtained from Ventana Medical Systems cannot be provided because this information is a proprietary information. The strength of CD117 expression was determined by using the criteria published by Sakurai et al [6].) A strength of $3+$ was considered the highest strength (i.e. strong positivity). Mutation analysis for $c$-kit and PDGFR $\alpha$ genes was performed by a commercial laboratory by using standard techniques.

Grading of GIST was done based criteria described elsewhere [9]. Briefly, high-risk GISTs are $>10 \mathrm{~cm}$ or are high grade (MIB-1-labeling index $=10 \%$ or tumor necrosis), intermediate-risk GISTs are $>5 \mathrm{~cm}$ to $10 \mathrm{~cm}$ and are low grade (MIB-1-labeling index of $<10 \%$ and no tumor necrosis), and low-risk GISTs are $5 \mathrm{~cm}$ or $<5 \mathrm{~cm}$ and are low grade.

\section{Case Report}

A 77-year-old Caucasian male was evaluated at our hospital for suspected melena and anemia. He had history of shortness of breath on exertion. No other significant personal or family history was reported. Esophagogastroduodenoscopy showed a diffusely erythematous mucosa and an ovoid polypoid mass, which measured approximately $3 \mathrm{~cm}$ in maximum and was present in the center of lesser curvature of the stomach. The mass had a central ulceration. Small biopsy specimens were taken. There were two pieces of tan-colored firm tissue measuring 3 and $4 \mathrm{~mm}$ in maximum dimension. Microscopic examination showed epithelioid cells with mild pleomorphism, mild hyperchromasia, moderate nucleocytopasmic raio, no mitotes, no myxoid/chondroid or chordoid appearance, and strong and homogeneous positivity for CD117. A diagnosis of GIST was made. The surgeon laparoscopically removed the tumor which was located within the within the wall of the stomach with most of the tumor in the muscularis propria and submucosa and measured approximately $3.7 \mathrm{~cm} \times 2.5 \mathrm{~cm} \times 2.4 \mathrm{~cm}$ in size. Its cut surface was myxoid, tan-solid and brownish and the tumor was confined to the wall of the stomach (Figure 1). Microscopically, the tumor was present in the submucosa and muscularis propria, was non-encapsulated, was well circumscribed in the superficial part of the tumor, and was infiltrating the muscularis propria in the deeper part. The most of the tumor was composed of epithelioid cells that were surrounded by a myxoid and fibrous stroma in such a way that several zones of chondroid-like change were observed (Figure 2). In other areas, the tumor cells resembled physaliferous cells surrounded by a myxoid stroma and, therefore, looked chordomatous (Figures 3). Almost all of the tumor showed myxoid change, but approximately $75 \%$ it showed an admixture of myxoid change and chondorod zones, while the rest of it was a combination of myxoid and chordoid areas. Extremely rare mitotic figures (about 1 in 100 high power fields) could be found. No tumor necrosis was seen. Increased blood vessel proliferation was noted in many areas. Collagenous fibrosis and hyaline change were also present in the stroma. Alcian blue stain highlighted the myxoid change (Figure 4), trichrome stain confirmed the collagenous fibrosis and CD34 highlighted numerous blood vessels. The toluidine blue stain and the CD117 immunohistochemical stain showed very rare mast cells in the stroma of the neoplasm. The neoplastic cells were strongly and homogeneously positive for CD117 (Figure 5), PDGFR (Figure 6), DOG.1, and CD99. CD34, smooth muscle actin, desmin, and S-100 were negative in the neoplastic cells. Immunohistochemical negativity for epithelial membrane antigen (EMA) as well as pancytokeratin ruled-out chordomatous differentiation, and negativity for D2-40 ruled-out chondsarcomatous differentiation. The GIST was graded as low-risk. No mutations were detected in the $c$-kit gene exons $8,9,11,13$, and 17 and no mutations were detected in the PDGFR $\alpha$ gene exons 12 and 18. Margins of resection were free of tumor. PET/CT scan showed showed no other mass lesions anywhere in the body. No further treatment was needed. The patient has been free of symptoms for last 10 months after his surgery.

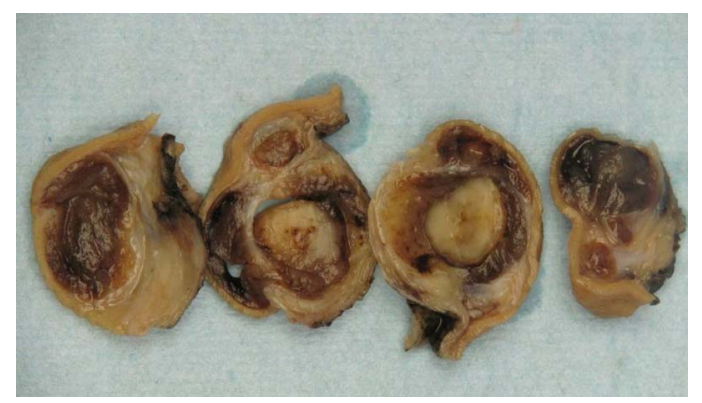

Figure 1. Cut surface of gastric tumor shows variegated appearance created by solid-tan, myxoid and brownish areas. 


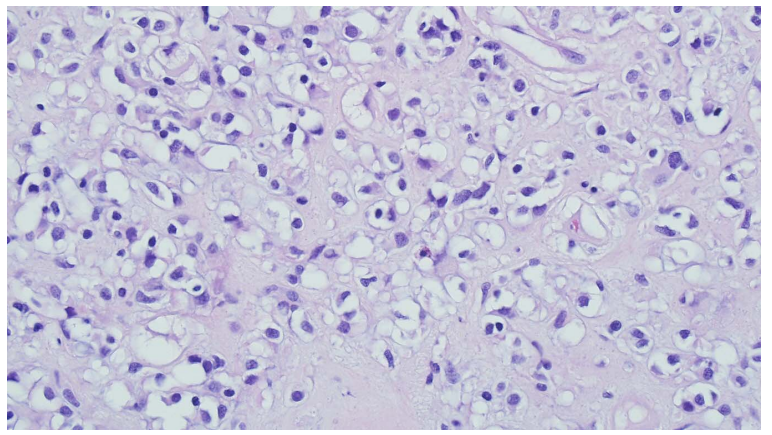

Figure 2. Chondroid-looking areas with fibrous stoma admixed with a myxoid change (H \& E Stain; ×400).

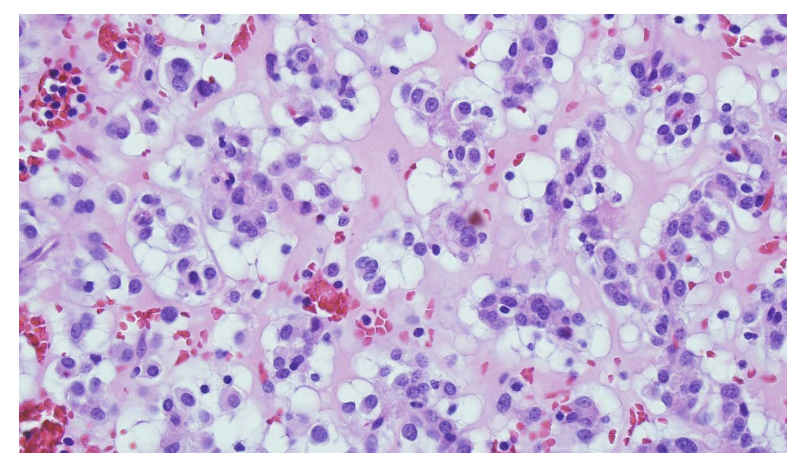

Figure 3. Chordoid-looking areas showing physaliferous cell-like cells and myxoid change ( $\mathrm{H} \& \mathrm{E}$ Stain; $\times 400)$.

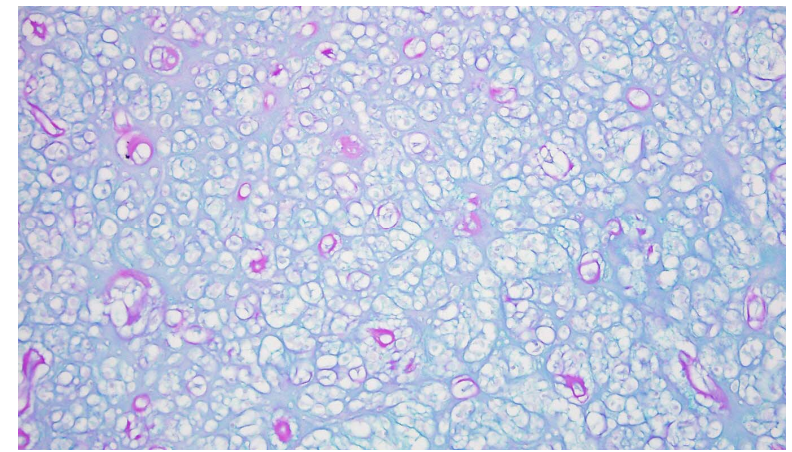

Figure 4. Alcian blue-PAS stain highlighting myxoid change (H and E Stain; $\times 200)$.

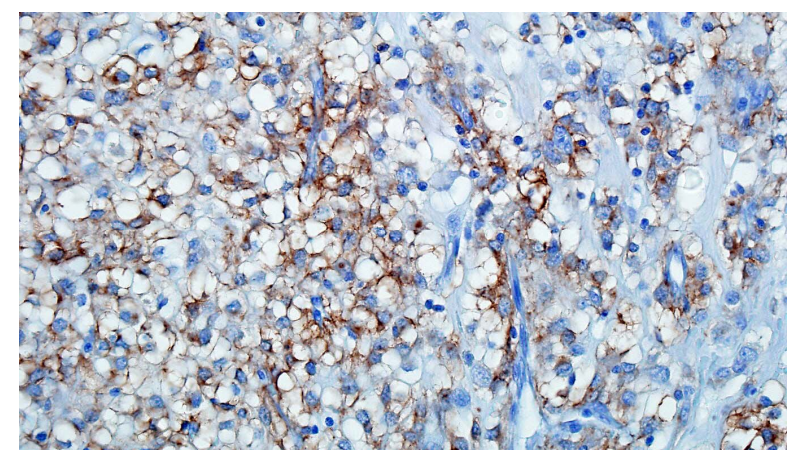

Figure 5. CD117 positivity in the chondroid and chordoid areas (Immunohistochemical Stain; $\times 400)$.

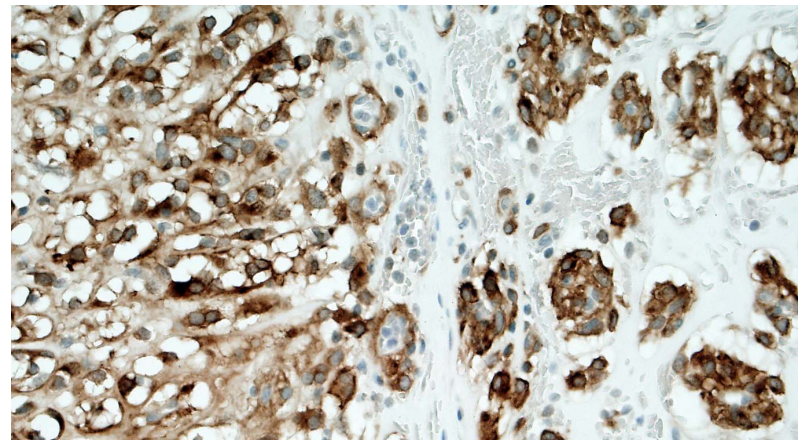

Figure 6. PDGFR $\alpha$ positivity in the chondroid and chordoid areas (Immunohistochemical Stain; $\times 400)$.

\section{Comment}

Our case is very peculiar because of its histological resemblance to an extraskeletal myxoid chondrosarcoma due a myxoid/chondroid/chordoid change, a strong expression of CD117 and lack of mutations in the c-kit gene (exons 8, 9,11,13, and 17) and in the PDGFR $\alpha$ gene (exons 12 and 18). It is possible that our case does have a $c$-kit and/or PDGFR $\alpha$ mutation that has not been recognized so far. In a recent study, myxoid change in epithelioid GISTs has been associated with lack or weakness of CD117 immunohistochemical positivity, lack of c-kit mutations, presence of platelet-derived growth factor recptor alpha (PDGFR $\alpha)$ gene mutations, and good prognosis [7]. Our case showed strong CD117 positivity, lack of mutations both in both c-kit gene and PDGFR gene and is free of any symptoms during last 10 months after the resection of his tumor. Association of absence of $c$-kit gene mutations with presence of PDGFR $\alpha$ gene mutations encoding $P D G F R \alpha$ protein in GISTs and also with an effective response to imatinib mesylate has been reported recently in a few other studies [10-13]. A single case of epithelioid-cell predominant GIST mimicking chondrosarcoma due to chondro-myxoid change has been reported and it showed strong expression of CD117. No analysis for $c$-kit or PDGFR $\alpha$ mutations was done in this case [8]. We are reporting a case of myxoid epithelioid GIST with predominance of chondroid areas and chordoid areas that morphologically resembled chordoid sarcoma (extraskeletal myxoid chondrosarcoma) and showed strong immunohistochemical positivity for CD117, DOG. 1 and PDGFR $\alpha$. DOG.1 and PDGFR $\alpha$ positivity can be seen in CD117-positive/c-kit positive GISTs $[8,14,15]$. GISTs showing immunohistochemical positivity for CD117 and PDGFR $\alpha$ can be negative for mutations in the c-kit and/or PDFGR $\alpha$ genes and can show clinical response to imatinib [16,17]. Our case showed positivity for CD117 and PDGFR a and lack of c-kit gene mutations and PDGFR $\alpha$ gene mutations. CD99 expression has been described in GIST $[8,18]$ and it was positive in our 
case. The significance of CD99 is not clear.

Based on the recently reported case of a myxoid epithelioid GIST with chondroid appearance [8] and our case of a myxoid epithelioid GIST with chondroid and chordoid appearance, it looks like that if we have chondroid appearance in a myxoid GIST we can predict CD117 positivity and, therefore, responsiveness to imatinib mesylate. Our case is the first of its kind where a myxoid epithelioid GIST shows chondroid and chordoid areas mimicking chordoid sarcoma (extraskeletal myxoid chondrosarcoma). We also raise the possibility of association of strong CD117 positivity with myxoid epithelioid GISTs if they have chondroid/chordoid appearance. A larger study can help confirm a relationship of strong CD117 positivity with a myxoid epithelioid GIST with chondroid/chordoid appearance.

In the two studies about myxoid GIST (without chondroid or chordoid appearance), 23 of 27 cases were followed-up for 1 month to 8 years and others were lost to follow-up at the time of publication of these studies. None of the followed-up patients died of GIST-related cause, but the follow-up period was less than 5 years in 14 of 23 cases at the time of publication [6,7]. Prognostic significance of chondroid/chordoid appearance in a myxoid GIST is difficult to assess because of the rarity of reported cases in the literature. One case of gastric myxoid GIST with chondroid appearance was considered to be an indolent neoplasm without any comment about follow-up of the case [8]. Our case is alive and well about 10 months after the resection of his gastric GIST with chondroid and chordoid appearance, but the followup is not long enough to determine its long-term clinical behavior.

\section{REFERENCES}

[1] M. Meittinen, M. Sarlomo-Rikala and J. Lasota, "Gastrointestinal Stromal Tumors," Annales Chirurgiae et Gynaecologiae Fenniae, Vol. 87, No. 4, 1998, pp. 278281.

[2] S. R. Hamilton and L. A. Aaltonen, "World Health Organization Classification of Tumors: Pathology and Genetics of Tumors of the Digestive System," IARC Press, Lyon, 2000.

[3] L. G. Kindblom, H. E. Remotti, F. Aldenborg and J. M. Meis-Kindblom, "Gastrointestinal Pacemaker Cell Tumor (GIPACT): Gastrointestinal Stromal Tumors Show Phenotypic Characteristics of the Interstitial Cells of Cajal," American Journal of Pathology, Vol. 152, No. 5, 1998, pp. 1259-1269.

[4] C. D. Fletcher, J. J. Berman, C. Corless, et al., "Diagnosis of Gastrointestinal Stromal Tumors: A Consensus Approach," Human Pathology, Vol. 33, No. 5, 2002, pp. 459465. doi:10.1053/hupa.2002.123545

[5] E. Wardelmann, I. Neidt, E. Bierhoff, et al., "c-kit Muta- tions in Gastrointestinal Stromal Tumors Occur Preferentially in the Spindle Rather than in the Epitheiloid Cell Variant," Modern Pathology, Vol. 15, No. 2, 2002, pp. 125-136. doi:10.1038/modpathol.3880504

[6] S. Suster, D. Sorace and A. Moran, "Gastrointestinal Stromal Tumours with Prominent Myxoid Matrix. Clinicopathologic, Immunohistochemical and Ultrastructural Study of Nine Cases of a Distinctive Morphologic Variant of Myogenic Stromal Tumor," American Journal of Surgical Pathology, Vol. 19, No. 1, 1995, pp. 59-70. doi:10.1097/00000478-199501000-00008

[7] S. Sakurai, T. Hasegawa, Y. Sakuma, et al., "Myxoid Epithelioid Gastrointestinal Stromal Tumor (GIST) with Mast Cell Infiltrations: A Subtype of GIST with Mutations of Platelet-Derived Growth Factor Receptor Alpha Gene," Human Pathology, Vol. 35, No. 10, 2004, pp. 1223-1230. doi:10.1016/j.humpath.2004.07.008

[8] A. Rajput, C. Levea, M. Intengan, J. Kane and J. F. Gibbs, "Gastrointestinal Stromal Tumor: Chondro-Myxoid Variant Mimicking Chondrosarcoma," Sarcoma, Vol. 91, No. 1-2, 2005, pp. 25-28. doi: $10.1080 / 13577140500043708$

[9] T. Hasegawa, Y. Matsuno, T. Shimoda, et al., "Gastrointestinal Stromal Tumor: Consistent CD117 Immunostaining for Diagnosis, and Prognostic Classification Based on Tumor Size and MIB-1 Grade," Human Pathology, Vol. 33, No. 6, 2002, pp. 669-676. doi:10.1053/hupa.2002.124116

[10] M. R. Peterson, Z. Piao, N. Weidner and E. S. Yi, "Strong PDGFRA Positivity Is Seen in GISTs but Not in Other Intrabdominal Mesenchymal Tumors: Immunohistochemical and Mutational Analyses," Applied Immunohistochemistry \& Molecular Morphology, Vol. 14, No. 4, 2006, pp. 390-396. doi:10.1097/01.pai.0000203038.33414.a3

[11] M. C. Heinrich, C. L. Corles, A. Duensing, et al., "PDGFRA Activating Mutations in Gastrointestinal Stromal Tumors," Science, Vol. 299, No. 5607, 2003, pp. 708-710. doi:10.1126/science. 1079666

[12] S. Hirota, A. Obashi, T. Nishida, et al., "Gain-of-Function Mutations of Platelet-Derived Growth Factor Receptor Alpha Gene in Gastrointestinal Stromal Tumors," Gastroenterology, Vol. 125, No. 3, 2003, pp. 660-667. doi:10.1016/S0016-5085(03)01046-1

[13] M. C. Heinrich, C. L. Corless, G. D. Demetri, et al., "Kinase Mutations and Imatinib Response in Patients with Metastatic Gastrointestinal Stromal Tumor," Journal of Clinical Oncology, Vol. 21, No. 23, 2003, pp. 4342-4349. doi:10.1200/JCO.2003.04.190

[14] G. H. Kang, A. Srivastava, Y. E. Kim, et al., "DOG1 and PKCӨ Are Useful in the Diagnosis of KIT-Negative Gastrointestinal Stromal Tumors," Modern Pathology, in Press.

[15] M. Novelli, S. Rossi, M. Rodriguez-Justo, et al., "DOG1 and CD117 Are the Antibodies of Choice in the Diagnosis of Gastrointestinal Stromal Tumors," Histopathology, Vol. 57, No. 2, 2010, pp. 259-270. doi:10.1111/j.1365-2559.2010.03624.x

[16] Y. Jiang, L. Ming, A. J. Montero, E. Kimchi, M. Nikfar- 
jam and K. F. Staveley-O'Carroll, "Optimizing Imatinib Mesylate Treatment in Gastrointestinal Stroma Tumors," Gastrointestinal Cancer Research, Vol. 2, No. 5, 2008, pp. 245-250.

[17] Y. Koh, H. Kim, H. Lee, D. Oh, J. Kim, S. Im, et al., "KIT and PDGFRA Mutation Status and Their Immunohistochemical (IHC) Expression Profile of Gastrointestinal Stromal Tumor (GIST) Patients with Imatinib (IMT):
Seven-Year Single-Center Experience," Journal of Clinical Oncology, Vol. 27, No. 15, 2009, p. 27.

[18] V. B. Shidham, M. Chivukula, D. Gupta, R. N. Rao and R. Komorowski, "Immunohistochemical Comparison of Gastrointestinal Stromal Tumor and Solitary Fibrous Tumor," Archives of Pathology \& Laboratory Medicine, Vol. 126, No. 10, 2002, pp. 1189-1192. 